Para enlazar con este artículo / To link to this article:

http://dx.doi.org/10.6035/MonTI.2019.ne5.7

Para citar este artículo / To cite this article:

Andrade Vargas, Lucy; Margoth Iriarte Solano; Diana Rivera-Rogel; Isidro Marín-Gutiérrez \& Andrea Velásquez Benavides. (2019) "Competencias mediáticas de estudiantes de bachillerato en Ecuador frente al currículo escolar." En: Montero Küpper, Silvia; Iván Puentes Rivera \& Montserrat Vázquez Gestal (eds.) 2019. Comunicación, Traducción e Interpretación / Communication, Translation and Interpreting. MonTI Special Issue 5, pp. 166-186.

\title{
COMPETENCIAS MEDIÁTICAS DE ESTUDIANTES DE BACHILLERATO EN ECUADOR FRENTE AL CURRÍCULO ESCOLAR
}

\section{MEDIA LITERACY OF HIGH SCHOOL STUDENTS IN ECUADOR AGAINST THE SCHOOL CURRICULUM}

\author{
LUCY ANDRADE VARGAS \\ ldandrade@utpl.edu.ec \\ Universidad Técnica Particular de Loja \\ MARGOTH IRIARTE SOLANO \\ miriarte@utpl.edu.ec \\ Universidad Técnica Particular de Loja \\ DIANA RIVERA-ROGEL \\ derivera@utpl.edu.ec \\ Universidad Técnica Particular de Loja \\ ISIDRO MARÍN-GUTIÉRREZ \\ imarin@utpl.edu.ec \\ Universidad Técnica Particular de Loja \\ ANDREA VELÁSQUEZ BENAVIDES \\ avvelasquez@utpl.edu.ec \\ Universidad Técnica Particular de Loja
}

Este obra está bajo una licencia de Creative Commons Reconocimiento 4.0 Internacional. 


\title{
Resumen
}

El desarrollo tecnológico de la sociedad actual demanda hoy en día que la competencia mediática del alumnado sea concebida como una variable más en el diseño curricular para solventar las necesidades diarias de conocimiento de los medios. Esta afirmación parte del diagnóstico de los niveles de competencia mediática del alumnado de bachillerato en Ecuador. Se realizó un estudio cualitativo de análisis de contenido en el currículo de bachillerato siguiendo las dimensiones del concepto de competencia mediática de Ferrés y Piscitelli. El estudio determinó que el 8\% del total de las destrezas contempladas en el bachillerato contribuyen al desarrollo de las competencias mediáticas, mientras que el $92 \%$ no lo hace. Se prueba con ello que el porcentaje es bajo, frente al total de destrezas trabajadas, limitando con esto la capacidad de los estudiantes para percibir, analizar, discriminar y disfrutar de la comunicación mediática.

Palabras clave: Ecuador; Curriculum; Competencias mediáticas; Educomunicación; Bachillerato; TIC

\begin{abstract}
This article is based on the diagnosis made of the competence levels of the Baccalaureate students in Ecuador in relation to the media. A qualitative study of content analysis in the baccalaureate curriculum following the dimensions of the concept of media competence of Ferrés and Piscitelli was carried out. The study made it possible to determine that $8 \%$ of the total skills contemplated in the baccalaureate contribute to the development of media skills, while $92 \%$ do not. This proves that the percentage is low compared to the total of skills worked, limiting with this the ability of students to perceive, analyze, discriminate and enjoy media communication.
\end{abstract}

Keywords: Ecuador; Curriculum; Media literacy; Media education; High school; ICT

\section{Introducción}

\subsection{Las competencias mediáticas en el contexto ecuatoriano}

Inicialmente se desarrolló el concepto de competencia en el mundo laboral y de la empresa. Poco a poco, se fue introduciendo en el mundo escolar y académico hasta llegar a ser un eje temático en las recientes reformas educativas, tanto escolares como universitarias, en la mayor parte de los países europeos y latinoamericanos. Entendemos por competencia la unión de conocimientos, 
destrezas y actitudes que son necesarios para un determinado contexto. Así, las competencias mediáticas deben desarrollar la autonomía de las personas y comprometerlas tanto social como culturalmente (Ferrés \& Piscitelli 2012).

La competencia, además de un saber hacer, debe incluir el saber entender, comprender las implicaciones de los hechos, prever las consecuencias y asumirlas de manera responsable. En Ecuador, los medios de comunicación social, tanto públicos como privados o comunitarios, deben satisfacer las necesidades de información, educación y entretenimiento de todos los grupos sociales (UNESCO 2011: 95). Los medios audiovisuales de Ecuador no tienen ningún tratamiento especial para personas no alfabetizadas. En la Ley Orgánica de Comunicación aprobada en 2013, en su artículo 74 sobre responsabilidades comunes aparece en su apartado 9: "Propender a la educomunicación". Pero ¿se está desarrollando este apartado?

Se está trabajando en programas de uso de TIC en Ecuador en los últimos años; uno de ellos es el fortalecimiento del aprendizaje basado en uso de TIC (Peñaherrera 2011). La primera iniciativa estatal en Ecuador cuyo fin era incorporar las TIC en el sistema educativo comenzó en 2002, cuando se dotó a los maestros de ordenadores implementados con un programa de capacitación destinado al uso pedagógico. Se trataba del programa Maestr@.com (Peñaherrera 2012). Posteriormente, hubo un estancamiento por la situación política. En 2006 se afianzó la incorporación de las TIC a través del Libro Blanco de la Sociedad de la Información, que constituyó el marco de la política sobre TIC (CONATEL 2006).

Así que, la integración de las TIC en el sector educativo en Ecuador está obteniendo infraestructuras, aulas con ordenadores, recursos informáticos, software educativo, formación al profesorado, creación de portales educativos y soporte técnico. Existen diversas publicaciones sobre los estándares educativos en TIC (Ministerio de Educación de Ecuador 2012). También se han realizado estudios sobre el uso de TIC en docentes ecuatorianos, en donde es necesario un plan de formación que los implique para adquirir competencias digitales. Es importante tanto la formación como la autoformación (Valdivieso, 2010). Encontramos que en Ecuador la práctica de incorporación de las TIC se realiza según las iniciativas de investigadores y académicos (Marín-Gutiérrez, Díaz-Pareja \& Aguaded 2013). 
En Ecuador se ha intentado dar una visión del significado de competencia mediática y ver qué se ha hecho hasta ahora en el contexto escolar ecuatoriano. Principalmente, se han realizado investigaciones sobre competencias mediáticas en el bachillerato en el sur de Ecuador (Marín-Gutiérrez et al. 2014). Los resultados muestran que son los estudiantes quienes han aprovechado de mejor manera el uso de las TIC, en procesos de tinte social, mas no académico-formativo, puesto que en el contexto escolar existen limitaciones de los docentes para utilizar las tecnologías. De tal forma, se sugiere considerar en el campo académico y del currículo oficial el uso obligatorio de las TIC como herramientas que potencien el aprendizaje e interacción entre profesores y estudiantes.

\subsection{Las competencias mediáticas en el currículo del bachillerato ecuatoriano}

El término "currículo" implica varias acepciones. Algunos autores como Posner (2003) consideran que "el currículo es un plan de construcción (y formación) que se inspira en conceptos articulados y sistemáticos de la pedagogía y otras ciencias sociales afines que pueden ejecutarse en un proceso efectivo y real llamado enseñanza" (Posner 2003). Para Lafrancesco (2004), el "currículo" es el conjunto de objetivos de aprendizaje operativos, convenientemente agrupados en unidades funcionales y estructurales de tal manera que conduzcan a los estudiantes a alcanzar un grado de dominio, que normen, eficientemente, las actividades de enseñanza-aprendizaje.

El "currículo" entonces sistematiza y organiza coherentemente los objetivos, métodos, recursos, conocimientos, procesos de enseñanza-aprendizaje y criterios de evaluación. Ahora bien, la sociedad del conocimiento exige, por un lado, nuevos perfiles en los estudiantes y, por otro, "contemplar de forma prioritaria en los currículos el uso de las TIC" (Acosta 2010). Surge entonces la pregunta: ¿cómo integrar competencias mediáticas en el currículo de bachillerato ecuatoriano? Este es un tema aparentemente contemplado en el currículo y aplicado en los procesos de enseñanza-aprendizaje. Sin embargo, la incorporación va más allá. Requiere de la estructuración de verdaderas comunidades de aprendizaje que manejen la información, aprendan con ella, la procesen y la produzcan para ser transmitida, revalorada, estudiada, como un ciclo en permanente movimiento (Abendaño \& Parada 2012). 
De ahí la importancia de incluir en el currículo escolar destrezas que promuevan el desarrollo del pensamiento lógico, crítico, creativo, productivo y significativo a través del uso de los medios de comunicación y TIC, a fin de consolidar habilidades que permitan al alumnado comunicarse de manera efectiva, buscar información, visualizar y objetivar lugares, hechos, procesos o situaciones de la realidad (MEE 2014). En otras palabras, saber decir y saber interpretar la información que tienen a su alcance, generando investigación, creatividad e innovación (Abendaño \& Parada 2012) para la resolución de problemas cotidianos y lograr así la formación integral del estudiante en el marco del Buen Vivir.

El bachillerato ecuatoriano propone una estructura curricular que parte de un tronco común de aprendizajes básicos en el primero y segundo año; en el tercer año, aunque se conservan 20 horas semanales de asignaturas comunes, se abren opciones diversas de estudio. Durante los tres años de duración de este nivel, todos los estudiantes deben cursar el grupo de asignaturas generales definidas en el currículo nacional obligatorio, pudiendo complementarse de acuerdo con las especificidades y particularidades de las instituciones educativas (MEE 2011).

Esta modalidad busca, entre otros aspectos, que el estudiante utilice como perfil de salida las herramientas tecnológicas de forma reflexiva y pragmática para comprender la realidad circundante, resolver problemas, tener acceso a la sociedad de la información y manifestar su creatividad, evitando la apropiación y uso indebido de la información (González 2010).

Con el fin de alcanzar esta proyección se propone un currículo basado en destrezas con criterio de desempeño, las que expresan el saber hacer, con una o más acciones integrales que establecen relaciones con un determinado conocimiento, y con diferentes grados de complejidad, según condicionantes de rigor científico-cultural, espaciales y temporales, entre otros (MEE 2014). El desarrollo de las destrezas serán medidas por indicadores de evaluación expresados especialmente en las cuatro áreas del currículo nacional: matemática, lengua y literatura, ciencias naturales y estudios sociales, aclarándose que, en el futuro, se formularán estándares correspondientes a otras áreas de aprendizaje, tales como TIC, lengua extranjera, formación ciudadana, educación artística y educación física (MEE 2011). 
Frente a estos requerimientos, en el currículo del bachillerato ecuatoriano de 2011 se plantean varias destrezas que apuntan a la incorporación de competencias mediáticas para el empleo en los procesos de enseñanza-aprendizaje. Sin embargo, sigue siendo necesaria la incorporación de mediadores tecnológicos en todas las asignaturas para que apoyen los procesos de aprendizaje colaborativo, faciliten la interacción permanente y conviertan a las instituciones tradicionales en nodos de un entramado de red que puede proporcionar entornos de comunicación sincrónica y asincrónica. Así, se verá reforzado el diálogo, y se proporcionará a la vez una simulación que mejore los resultados de la clase tradicional (Montero et al. 2008). La apropiación de las TIC por parte del docente favorece la interacción de estas herramientas con el conocimiento y permite un desarrollo de estructuras mentales en los estudiantes y los prepara integralmente para la vida a través de la convivencia y participación activa en una sociedad intercultural y plurinacional (Riascos, Quintero \& Ávila 2009).

\section{Metodología}

La investigación se plantea conocer y explicar una realidad concreta y determinar ciertas generalizaciones que puedan explicar qué dimensión educomunicativa se encuentra en los currículos de bachillerato de Ecuador. Estos objetivos nos llevan a recurrir a una metodología empírico-analítica y a un diseño no experimental y descriptivo, de acuerdo con lo establecido por Cáceres (2008) y Corbetta (2003).

Las fases que se han seguido en este proceso fueron: el planteamiento del problema de investigación, la delimitación de los objetivos de estudio, la formulación de hipótesis, la identificación y denominación de las distintas dimensiones que se deberán estudiar, el planteamiento de la investigación, el instrumento de recogida de información y las técnicas de análisis de datos cualitativos con Atlas.Ti. Las hipótesis que se propusieron fueron: (H:1) El porcentaje de destrezas que incluyen competencias mediáticas es bajo en relación al porcentaje global de destrezas propuestas en el currículo de bachillerato; (H:2) Las dimensiones que constituyen la competencia mediática se abordan en el currículo con la misma frecuencia; (H:3) Las dimensiones de 
las competencias mediáticas, en las destrezas del currículo, predominan de acuerdo a las particularidades de la asignatura.

\section{Método}

El desarrollo de la investigación priorizó como método el análisis de contenido. Esta metodología, según Bardin (1991) y López (2002), es de tipo descriptiva y pretende extraer de un contenido los componentes básicos de un fenómeno determinado.

\section{Unidades de análisis}

En este estudio, la unidad de análisis se configuró de la siguiente manera: en primer lugar, una unidad de registro de base gramatical constituida por frases y párrafos derivados de las competencias mediáticas en sus niveles de análisis y expresión; en segundo lugar, una unidad de contexto conformada por textos legales del Ministerio de Educación y, en tercer lugar, la existencia o no de las categorías presentes en cada una de las destrezas con criterio de desempeño, así como la cuantificación de su presencia.

Los materiales empleados fueron los ocho textos curriculares que se desarrollan en el nivel de bachillerato, de los cuales se analizaron 27 asignaturas correspondientes a 11 áreas: Ciencias experimentales, Ciencias sociales, Economía, Educación artística, Emprendimiento, Filosofía, Informática aplicada a la educación, Investigación de ciencia y tecnología, Lengua y literatura, Matemáticas y Psicología; de este modo, la generalización de los resultados se extendía al currículo de bachillerato. En el contexto de Ecuador, al finalizar el bachillerato se aplican las pruebas "Ser bachiller" como requisito para acceder a un cupo en la universidad, y se evalúa el grado en que los estudiantes alcanzan diferentes destrezas, entre las que no aparece la competencia mediática; de ahí que se justifique la investigación en este nivel. La población objeto de estudio ha sido 978 unidades de registro (destrezas con criterio de desempeño), de las cuales 82 correspondían a las competencias mediáticas.

\section{Procedimiento}

Los documentos curriculares se obtuvieron de las páginas web oficiales del Ministerio de Educación de Ecuador para asegurar la revisión desde la fuente original. Los criterios de selección fueron: la Ley Orgánica de Educación 
Intercultural (2011) y los Lineamientos Curriculares para el Bachillerato General Unificado de Ecuador (2011). La fecha de búsqueda tuvo lugar en los meses de mayo, junio y julio de 2015.

\section{Análisis de la información}

Previo al análisis de la información se realizó la codificación de las unidades de registro (destrezas con criterio de desempeño) atendiendo a los lineamientos curriculares de Ecuador, al año, área y asignatura del bachillerato; se establecieron criterios de búsqueda para identificar las destrezas con criterio de desempeño asociadas a las competencias mediáticas de acuerdo con las dimensiones, subdimensiones y descriptores establecidos por Ferrés (2006, 2007). Seguidamente se efectuó el análisis de las destrezas de las siguientes asignaturas: Biología superior, Química superior, Física superior, Biología, Física, Química, Física, química, Educación para la ciudadanía, Problemas del mundo contemporáneo, Sociología, Historia y ciencias sociales, Corrientes filosóficas, Desarrollo del pensamiento filosófico, Informática aplicada a la educación, Investigación ciencia y tecnología, Lectura crítica de mensajes, Lengua y literatura, Lengua y cultura ancestral, Redacción creativa, Psicología, Economía, Apreciación musical, Teatro, Educación artística, Emprendimiento y gestión, Matemática y Matemática superior.

Estas unidades de registro fueron introducidas en el programa ATLAS. ti y se fueron categorizando en función de los 55 descriptores establecidos por Ferrés y Piscitelli (2012) para las seis dimensiones que definen la competencia mediática: lenguajes, tecnología, procesos de interacción, procesos de producción y difusión, ideología y valores y estética.

La dimensión de "Lenguaje" se relaciona con el conocimiento de los códigos que hacen posible el lenguaje audiovisual y la capacidad de utilizarlos para comunicarse de manera sencilla pero efectiva. La dimensión de "Tecnología" tiene que ver con el conocimiento teórico del funcionamiento de las herramientas que hacen posible la comunicación audiovisual para entender cómo son elaborados los mensajes y la capacidad de usar las herramientas menores, más sencillas, para establecer una comunicación eficiente en el entorno audiovisual. La dimensión de "Procesos de producción y programación" es el "conocimiento de las funciones y tareas asignadas a los 
principales agentes de producción y las fases en las que se descomponen los procesos de producción y programación" (Ferrés, 2017), y toma en cuenta la elaboración de mensajes audiovisuales, es decir, la capacidad de producción de la persona y la adquisición de conocimientos sobre la importancia de los nuevos ambientes para comunicar. La dimensión de "la ideología y valores" es la capacidad comprensiva y crítica de los mensajes audiovisuales en cuanto representaciones y análisis crítico de la realidad y, en consecuencia, como portadores de ideología y de valores. La dimensión de "Recepción" desarrolla la capacidad de reconocerse como audiencia activa, especialmente a partir del uso de las tecnologías digitales, que permiten la participación y la interactividad. La última dimensión es la "Estética", que analiza y valora los mensajes desde el punto de vista de la innovación formal y temática y la educación del sentido estético.

Finalmente, con los resultados generados se realiza el análisis descriptivo donde se identifica cuáles son las destrezas del currículo que contribuyen al desarrollo de las competencias mediáticas de los estudiantes de bachillerato.

\section{Resultados}

El estudio permitió determinar que el 8\% del total de las destrezas contempladas en el bachillerato contribuyen al desarrollo de las competencias mediáticas, mientras que el $92 \%$ no lo hace. Se prueba con ello que el porcentaje es bajo frente al total de destrezas trabajadas, lo que limita la capacidad de la persona para percibir, analizar, discriminar y disfrutar de la comunicación mediática. Es necesario, entonces, comprender la importancia de la inclusión de este tipo de competencias en el currículo. Nos encontramos en una sociedad digitalizada en donde "la profusión de medios, el avance y progreso tecnológico, las brechas intergeneracionales y la tan traída y llevada metáfora de los nativos digitales" (Prensky, 2001) generan la imperiosa necesidad de incluir y dinamizar destrezas enfocadas, entre otros aspectos, al desarrollo de las competencias mediáticas. Desde este análisis podemos concluir que el porcentaje de destrezas que incluyen competencias mediáticas es bajo en relación al porcentaje global de destrezas propuestas en el currículo de bachillerato, tal como se muestra en la Tabla 1. 
Tabla 1. Destrezas que desarrollan competencias mediáticas

\begin{tabular}{|l|c|c|}
\hline \multicolumn{1}{|c|}{ DESTREZAS } & F & $\%$ \\
\hline No implica competencias mediáticas & 896 & 91,65 \\
\hline Sí implica competencias mediáticas & 82 & 8,35 \\
\hline TOTAL & 982 & $100 \%$ \\
\hline
\end{tabular}

Fuente: Elaboración propia

En el Gráfico 1 se puede identificar que las dimensiones de lenguaje y tecnología se abordan mayoritariamente con un $39,2 \%$ y $31,71 \%$, respectivamente. Le siguen en representación la dimensión de ideología y valores con el 15,85\%, la dimensión de programación y producción con un 9,76\% y la dimensión de estética con un 3,66\%.

Esta clasificación permite ver que el currículo de bachillerato prioriza el desarrollo del conocimiento de códigos audiovisuales que hacen posible el lenguaje y la comunicación para el análisis de los mensajes y la perspectiva del sentido y la significación. Asimismo, también le da una mayor importancia al conocimiento teórico del funcionamiento, comprensión y utilización de herramientas tecnológicas que hacen posible la comunicación audiovisual de manera eficaz.

La capacidad de lectura comprensiva y crítica de los mensajes audiovisuales en cuanto a representaciones de la realidad que se explicitan en el currículo de bachillerato requiere fortalecerse como expresión y soporte de los intereses, contradicciones e ideologías y valores de la sociedad. La participación e interactividad en el uso de las tecnologías digitales no se promueve con frecuencia en el currículo de bachillerato. Por lo tanto, la valoración crítica de los mensajes audiovisuales en cuanto a lo emotivo, racional y contextual no desarrolla una programación y producción efectiva.

El análisis y valoración de los mensajes audiovisuales desde el punto de vista estético, así como la relación de éstos con otras formas de manifestación mediática y artística son las destrezas que menos se desarrollan en el currículo. Desde este análisis se concluye que las dimensiones que constituyen la competencia mediática se abordan en el currículo con diferente frecuencia, dependiendo de las destrezas, área y año de bachillerato, fortaleciendo a unas en mayor proporción que a otras. 
Gráfico 1. Dimensiones de la competencia mediática que se abordan en el currículo

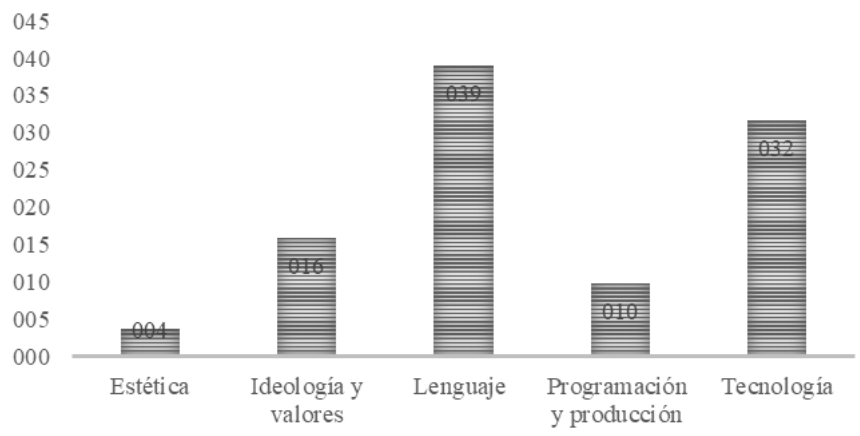

Fuente: Elaboración propia

Las dimensiones de lenguaje, tecnología, procesos de interacción, producción y análisis, ideología y valores y estética como ámbitos de la competencia mediática implican conceptos, procedimientos y actitudes que, desde nuestro punto de partida, tienen relación directa con las asignaturas y las destrezas que en estas se trabajan.

Así, en la asignatura de Educación Artística, el 100\% de las destrezas que se vinculan con las competencias mediáticas tienen relación con la dimensión estética. Se describe en este campo una destreza en la que se evidencia con mayor índice la implicación para manipular material sonoro concreto y virtual con el fin de improvisar ritmos musicales de diversas regiones del país. Asimismo, en esta asignatura, tal como se muestra en la Tabla 2, la dimensión de ideología y valores se desarrolla en un mayor porcentaje con un 53,85\%, pues se plantean, entre otras destrezas, la comprensión y utilización ética de las TIC en el aprendizaje y se desglosa en actividades que requieren análisis, tanto de la programación del poder mediático como de la interpretación y la valoración de producciones artísticas.

Además, las asignaturas que implican en sus destrezas competencias mediáticas relacionadas con la ideología y los valores son la informática aplicada a la educación y biología (15,38\%) y, en un menor porcentaje $(7,69 \%)$, las asignaturas de educación para la democracia y problemas del mundo contemporáneo. Ahora bien, si analizamos las características de las destrezas 
y de los contenidos que se abordan podemos concluir que la dimensión ideología y valores se relaciona con las asignaturas, pues en estas se prioriza al ser humano, la valoración de su entorno, las reflexiones, el conocimiento a partir de la investigación y la práctica de valores.

Tabla 2. Dimensión ideología y valores y su implicación con las destrezas de las asignaturas

\begin{tabular}{|l|c|}
\hline \multicolumn{1}{|c|}{ Asignaturas } & $\begin{array}{c}\text { Dimensión Ideología } \\
\text { y valores (\%) }\end{array}$ \\
\hline Educación artística & 53,85 \\
\hline Informática aplicada a la educación & 15,38 \\
\hline Educación para la ciudadanía & 7,69 \\
\hline Biología & 15,38 \\
\hline Problemas del mundo contemporáneo & 7,69 \\
\hline Total & 100 \\
\hline
\end{tabular}

Fuente: Elaboración propia

La tabla 3 muestra cómo la dimensión del lenguaje tiene una relación directa con la asignatura de lengua y literatura $(28,13 \%)$, pues esta considera prioritario en su enfoque la formación ciudadana y la democracia a través del desarrollo de competencias comunicativas de la lengua y del empleo de códigos que permiten la comunicación en sus diversos tipos. Es en esta asignatura, apoyada de la lectura crítica y redacción creativa, donde la dimensión del lenguaje encuentra su horizonte, puesto que se fomenta el conocimiento de los códigos y la capacidad de análisis de los mensajes audiovisuales desde la perspectiva del sentido y del significado.

Cabe mencionar que en esta dimensión se aglutinan una mayor cantidad de asignaturas por la propia particularidad del área y de su forma de abordarlo, además de convertirse en una asignatura específica, pues forma parte de un eje transversal en el cual todas las asignaturas confluyen en varios momentos y, por tanto, busca el paso constante de una simple descripción a una criticidad sociocultural evidenciada en la naturaleza propia de las asignaturas (biología, educación artística, lectura crítica, todas con un 15,63\%). 
Se puede apreciar también que, en los resultados de las asignaturas experimentales (Química, Física-Química, Química aplicada y Física superior, todas con un $3,13 \%$, la implicación de la dimensión es relativamente baja; sin embargo, es considerada como un medio eficaz dentro del proceso de enseñanza-aprendizaje.

Tabla 3. Dimensión lenguaje y su implicación con las destrezas de las asignaturas

\begin{tabular}{|l|c|}
\hline \multicolumn{1}{|c|}{ Asignaturas } & $\begin{array}{c}\text { Dimensión Lenguaje } \\
(\%)\end{array}$ \\
\hline Biología & 15,63 \\
\hline Educación Artística & 15,63 \\
\hline Química & 3,13 \\
\hline Física-Química & 3,13 \\
\hline Química Aplicada & 3,13 \\
\hline Lectura Crítica & 15,63 \\
\hline Lengua Redacción Creativa & 12,5 \\
\hline Lengua y Literatura & 28,13 \\
\hline Física Superior & 3,13 \\
\hline Total & 100 \\
\hline
\end{tabular}

Fuente: Elaboración propia

El Gráfico 2 muestra cómo la dimensión de la programación tiene un mayor porcentaje de acuerdo a los resultados en la asignatura de Educación artística $(62,5 \%)$, de tal forma que el objetivo de imaginar, crear, innovar y desarrollar una mente flexible se logra a través de procesos en los cuales los estudiantes puedan transferir conocimientos y vincularlos con su realidad, utilizando los medios de comunicación para documentar las relaciones sociales más importantes del entorno a través de la fotografía, el vídeo o el texto.

Frente a los resultados, también, es necesario precisar que las asignaturas que más se apoyan en la dimensión de la programación son la de química, con un $25 \%$, y la de biología, con un $12,5 \%$. En este caso, los principios, leyes, teorías y procedimientos, muchas de las veces resultan subjetivos; de ahí la 
necesidad de que se emplee en estas asignaturas recursos didáctico-tecnológicos para objetivar el contenido y simular procesos o situaciones cotidianas. Se puede deducir que, efectivamente, la dimensión de programación sí se relaciona con las asignaturas en las cuales se desarrollan destrezas que implican competencias mediáticas.

Gráfico 2. Dimensión programación y su implicación con las destrezas de las asignaturas

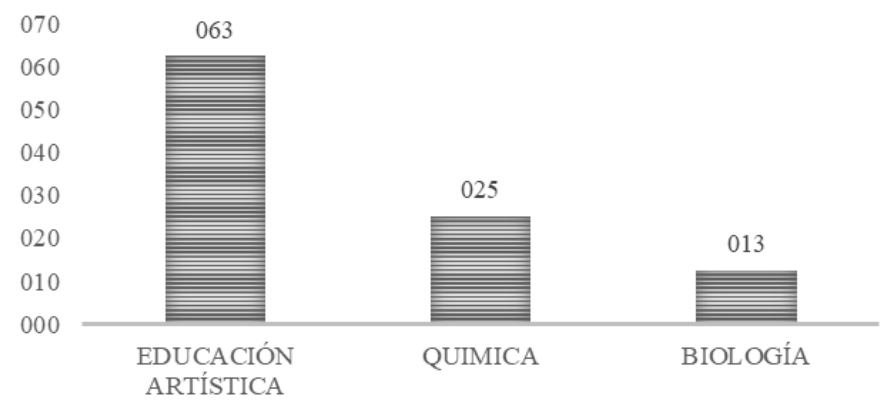

Fuente: Elaboración propia

Es necesario comentar que, en los lineamientos curriculares del bachillerato, la asignatura de informática aplicada a la educación no contempla destrezas con criterio de desempeño, de tal forma que, dada la relación de la denominación de la asignatura con las competencias mediáticas, se realizó un proceso inductivo de redacción de la destreza con criterio de desempeño a partir de los indicadores esenciales de evaluación.

En el caso de la dimensión tecnológica, tal como se muestra en el Gráfico 3 , la matemática es la asignatura que con mayor porcentaje $(76,92 \%)$ implica en sus destrezas esta dimensión; precisamente, un eje de aprendizaje del área contempla el uso de las tecnologías en la solución de problemas y, a decir del enfoque, las tecnologías se constituyen en herramientas no evaluables para la aplicación de conocimientos.

Las otras áreas que contemplan la dimensión tecnológica lo hacen en porcentajes menores, del 7,69\% (Educación artística) y del 3,85\% (Emprendimiento y gestión y Sociología). Estos datos nos permiten deducir 
que, si bien el área de matemática tiene mucha relación con la dimensión de la tecnología, se requiere que otras asignaturas impliquen también competencias mediáticas a fin de potenciar la relación.

Gráfico 3. Dimensión tecnológica y su implicación con las destrezas de las asignaturas

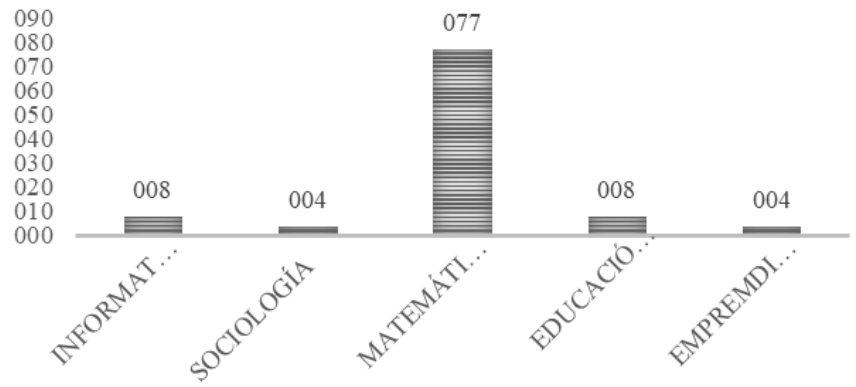

Fuente: Elaboración propia

\section{Discusión y conclusiones}

En la investigación que presentamos hemos constatado cómo están presentes en los currículos de bachillerato las seis dimensiones de las competencias mediáticas y, a grandes rasgos, vemos que unas están más desarrolladas que otras. Así, también el porcentaje de destrezas que incluyen competencias mediáticas es bajo en relación al porcentaje global de destrezas propuestas en el currículo de bachillerato; de igual manera se evidencia que las dimensiones de la competencia mediática no se abordan en el currículo con la misma frecuencia, ya que predominan de acuerdo a las particularidades de la asignatura, destacando especialmente las dimensiones de Lenguaje y Tecnología y, la menos destacada, la dimensión de la Estética, que se potenciaría a través de la música y las artes plásticas aplicando las TIC. Desde estas conclusiones, a continuación, se describen algunas líneas en las que se debe trabajar.

El currículo debe orientar el desarrollo de las capacidades cognitivas y afectivas que los estudiantes de bachillerato necesitan para desenvolverse en la sociedad de la información. Hay que valorar de forma crítica y selectiva los mensajes de los medios, reflexionar acerca del discurso mediático y tomar 
conciencia de su presencia en la propia vida personal, hábitos y actitudes, pues se tienen que interiorizar valores y acciones que permitan el desarrollo de la conciencia ciudadana global.

Es importante situar a la lectura en su forma más amplia dentro de las actividades de los docentes y en las destrezas del currículo. Es asimismo pertinente y factible incluir en la descripción de todas y cada una de las destrezas del currículo los recursos y estrategias tecnológico-didácticas que orienten el desarrollo de la competencia mediática como una forma de comunicación educativa afectiva y efectiva.

El sistema educativo ayudará a la comprensión del discurso de los medios de comunicación, tal y como afirma Bukingham (2007), no solo descodificando los mensajes, sino también entendiendo las ideologías y los valores de la sociedad actual en donde los jóvenes deben desarrollar sus capacidades creativas y de recepción crítica de los mensajes.

Se debe fomentar la inclusión de tablets y smartphones conectados a Internet para realizar actividades dentro del aula. Además, el alumnado debería recibir clases que les permitan relacionar la teoría con la práctica.

Por último, sería importante desarrollar una alfabetización mediática sistematizada en los centros de bachillerato integrados en el currículo.

\section{Referencias bibliográficas}

ABEndaño, William \& Abad Parada. (2012) "El currículo en la sociedad del conocimiento." Revista Educación y Educadores 11:1. Versión electrónica: $<$ https://goo.gl/G6ocTW>

ACOSTA, Luis. (2010) "El tratamiento de la información y competencia digital en la enseñanza aprendizaje en la historia en bachillerato." Catharum: Revista de ciencias y humanidades 11. Versión electrónica: <https://goo.gl/hq3wfo> BARDIN, Laurence. (1991) Análisis de contenido. Madrid. Ediciones Akal.

BuCKINGHAM, David. (2007) "Media education goes digital: an introduction." Learning, Media and Technology 32:2, pp. 111-119. Versión electrónica: <https://doi.org/10.1080/17439880701343006>

CÁCERES, Pablo. (2008) "Análisis cualitativo de contenido: Una alternativa metodológica alcanzable." Psicoperspectivas. Individuo y Sociedad 2:1, pp. 53-82. Versión electrónica: <https://goo.gl/tSWgZg> 
CONATEL. (2006) Libro Blanco. Estrategia para el desarrollo de la sociedad de la información en el Ecuador. Quito: CONATEL.

CORBETTA, Piergiorgio. (2003) Métodos y técnicas de investigación social. Madrid: McGraw Hill.

FERRÉS, Joan \& Alejandro Piscitelli. (2012) "La competencia en educación mediática: propuesta articulada de dimensiones e indicadores." Comunicar 38, pp. 75-82.

FERRÉS, Joan. (2007) "La competencia en comunicación audiovisual: dimensiones e indicadores." Comunicar 29, pp. 100-107. Versión electrónica: <https://goo. $\mathrm{gl} / \mathrm{D} 3 \mathrm{oSbd}>$

LAFRANCESCO, Giovanny. (2004) Currículo y plan de estudios. Estructura y planeamiento. Bogotá: Magisterio.

López Noguero, Fernando. (2002) "El análisis de contenido como método de investigación.” XXI Revista de Educación 4, pp. 169-179.

MARíN-GutiÉRrEZ, Isidro; Elena Díaz-Pareja \& Ignacio Aguaded. (2013) "La competencia mediática en niños y jóvenes: La visión de España y Ecuador." Chasqui 124, pp. 41-47.

MARÍN-GUTIÉRREZ, Isidro; Diana Rivera-Rogel \& Stephany Celly Alvarado. (2014) "Estudio sobre formación en competencia audiovisual de profesores y estudiantes en el sur de Ecuador." Cuadernos.Info 35, pp. 119-131. DOI: 10.7764/ cdi. 35.628

Ministerio de EdUCACIÓN DE ECUAdOR (MEE). (2011) Ley Orgánica de Educación Intercultural. Registro Oficial Ecuador, $2^{\circ}$ suplemento, 127.

Ministerio de EduCACión DE ECUAdor (MEE). (2012) Propuesta de estándares educativos. Versión electrónica: <https://goo.gl/oiv5fu>

Ministerio de EdUCACión De ECUAdor (MEE). (2014) Actualización y fortalecimiento curricular de la Educación General Básica. Quito: Ministerio de Educación del Ecuador.

MONTERO, Leyla; Jaime García \& Lida Rincón. (2008) "Una experiencia de aprendizaje incorporando ambientes digitales: competencias básicas para la vida." Revista Educación y Educadores 16:1. Versión electrónica: <https://goo.gl/ h3jiXg>

PeñAherRera-LeÓn, Mónica. (2011) "Evaluación de un programa de fortalecimiento del aprendizaje basado en el uso de las TIC en el contexto ecuatoriano." Revista Iberoamericana de Evaluación Educativa 4:2, pp. 72-91. Versión electrónica: <https://goo.gl/e2AtjX> 
Peñaherrera-León, Mónica. (2012) "Uso de TIC en escuelas públicas de Ecuador: análisis, reflexiones y valoraciones. EDUTEC." Revista Electrónica de Tecnología Educativa 40. Versión electrónica: <https://goo.gl/KmqnYj> POSNER, George. (2003) Docente del Siglo XXI. Cómo desarrollar una práctica docente competitiva. Análisis de Currículo. Bogotá: McGraw-Hill Interamericana.

PRENSKY, Marc. (2001) "Nativos digitales, inmigrantes digitales." On the horizon 9:6.

RiAsCoS, Sandra; Diana Quintero \& Gloria Ávila. (2009) "Las TIC en el aula: percepciones de los profesores universitarios." Educación y Educadores 12:3. Versión electrónica: <https://goo.gl/X7fHsn>

GONZÁlEZ, Rocío. (2010) "Tecnologías de la información y la comunicación, sociedad y educación. Sociedad, e-herramientas, profesorado y alumnado." RUSC. Universities and Knowledge Society Journal 7:2. Versión electrónica: <https://goo.gl/DD9kPG>

UnESCO. (2011) Análisis de Desarrollo Mediático en Ecuador 2011. Quito. UNESCO. VAldivieSO-Guerrero, Tania. (2010) "Uso de TIC en la práctica docente de los maestros de educación básica y bachillerato de la ciudad de Loja." Edutec 33. Versión electrónica: <https://goo.gl/3hrYCe>

\section{NOTAS BIOGRÁFICAS / BIONOTES}

Lucy Andrade VArgas es doctora en Desarrollo Psicológico, Familia, Educación e Intervención, Universidad Santiago de Compostela-España. Magíster en Pedagogía, Universidad Técnica Particular de Loja. Docenteinvestigadora de la UTPL, líneas y proyectos de investigación: Psicopedagogía: "Programa de formación docente para el desarrollo cognitivo. 2014- UNEDAECID-UTPL", "Intervención psicopedagógica para la mejora del clima social familiar y escolar. 2014-2017 UTPL", "Educación a padres de familia para su inclusión y ciudadanización. 2015-UTPL", Currículo y Didáctica: "Competencias mediáticas en jóvenes y padres de familia de instituciones educativas de Ecuador. 2014-2016-Ministerio de Economía y CompetitividadUniversidad de Huelva-UTPL" y "Rediseño curricular de las carreras de Ciencias de la Educación 2016-2017 UTPL". 
LuCy Andrade VArgas has a PhD in Psychological Development, Family, Education and Intervention, Universidad de Santiago de Compostela (Spain). Master in Pedagogy, Universidad Técnica Particular de Loja (Ecuador). Teacher-researcher of the UTPL, lines and research projects: Psychopedagogy: "Teacher training program for cognitive development. 2014- UNED-AECIDUTPL", "Psychopedagogical intervention for the improvement of the family and school social climate. 2014-2017 UTPL", "Education for parents for inclusion and citizenship. 2015-UTPL", Curriculum and Didactics: "Media competences in young people and parents of educational institutions in Ecuador. 2014-2016-Ministry of Economy and Competitiveness- University of Huelva-UTPL" and "Curriculum redesign of the Education Sciences careers 2016-2017 UTPL”.

MARGOTH IRIARTE SOLANO es magíster en Docencia y Evaluación Educativa. Coautora del libro La práctica Docente en la Educación Básica: experiencias y reflexiones, participante del proyecto "Competencias mediáticas en jóvenes, profesores y padres de familia de instituciones educativas públicas y privadas de Ecuador, práctica docente y calidad de aprendizajes en niñas, niños y adolescentes de Educación Básica en la región sur de Ecuador". Docente universitaria en pregrado y posgrado, en Educación Básica y Bachillerato.

MARGOTH IRIARTE SOLANO is a Master in Teaching and Educational Evaluation. Co-author of the book Teaching practice in Basic Education: Experiences and reflections. Participant in project "Media Competencies in young people, teachers and parents of public and private educational institutions of Ecuador, teaching practice and quality of learning in girls, boys and adolescents of Basic Education in the southern region of Ecuador. University Professor in Undergraduate and Postgraduate, in Basic Education and high school.

DiAna Rivera-Rogel es profesora titular de la Universidad Técnica Particular de Loja (UTPL), Ecuador. Académicamente es doctora en Comunicación y Periodismo y licenciada en Comunicación Social. Profesionalmente es directora del Área Sociohumanística de la UTPL. Coordinadora de la Red Interuniversitaria Euroamericana de Investigación sobre Competencias Mediáticas para la Ciudadanía (ALFAMED) en Ecuador. Coordinadora de la Red de Comunicación (REDU). Ha sido co-editora internacional de la revista científica Comunicar (2013-15). Ha publicado diversos artículos en 
revistas indexadas de impacto y capítulos de libros. Ha sido además miembro de consejos científicos, como el CIKI (2015), ISTI (2015), XESCOM (2016) y REDU (2016). Revisor de varias revistas científicas indexadas, tales como Comunicar, Cuadernos.info, Revista de Comunicación, Comunicación y Sociedad, Zer, entre otras.

DiAnA Rivera-Rogel is a full professor at the Universidad Técnica Particular de Loja (UTPL), Ecuador. PhD in Communication and Journalism and a degree in Social Communication. Director of the Área Sociohumanística of the UTPL, Coordinator of the Inter-University Network of Euro-American Research on Media Competencies for Citizenship (ALFAMED) in Ecuador. Coordinator of the Communication Network (REDU). She has been the international Co-editor of the scientific journal Comunicar (2013-15). She has published several articles in impact-indexed journals and book chapters. She has also been a member of scientific councils, such as CIKI (2015), ISTI (2015), XESCOM (2016) and REDU (2016). Reviewer of several indexed scientific journals, such as Comunicar, Cuadernos.info, Revista de Comunicación, Comunicación y Sociedad, Zer, among others.

ISIDRO MARÍn GutiÉRrez es licenciado en Ciencias Políticas y Sociología por la Universidad de Granada. Doctor (Ph.D.) en Antropología Social por la Universidad de Granada. Profesor Asociado de la Universidad de Huelva. Profesor-Tutor en el Centro Asociado de la UNED de Huelva. Investigador de la Universidad Técnica Particular de Loja. Su carrera investigadora está centrada principalmente en los consumos de drogas y las competencias mediáticas.

ISIDRO MARÍN GUTIÉRREZ holds a degree in Political Science and Sociology from the University of Granada. Ph.D. in Social Anthropology from the Universidad de Granada. Associate Professor at the Universidad de Huelva. Teacher-Tutor in the Associated Center of the UNED of Huelva. Researcher of the Private Technical University of Loja. His research career is mainly focused on drug consumption and media skills.

ANDREA VELÁSQUEZ BENAVIDES es profesora titular de Marketing y Publicidad de la Universidad Técnica Particular de Loja. Doctoranda en Comunicación e Industrias Creativas por la Universidad Santiago de Compostela-España y 
Máster en Comunicación e Industrias Creativas por la misma Universidad. Miembro del equipo de calidad de Comunicación Social y Relaciones Públicas. Actualmente investiga temas sobre consumo de medios, recepción, audiencias, marca digital y competencias mediáticas.

ANdREA VelÁsquez BENAVIDES is a Professor of Marketing and Advertising at the Universidad Técnica Particular de Loja. PhD in Communication and Creative Industries in the Universidad Santiago de Compostela (Spain) and Master in Communication and Creative Industries from the same University. Member of the quality team of Social Communication and Public Relations. She is currently investigating topics such as media consumption, reception, audiences, digital brands and media literacy. 